\title{
Sliding Observer for Induction Machines
}

\author{
Wei-Song Lin \& Chi-Hsiang Wang \\ Institute of Electrical Engineering National Taiwan University \\ 1, Sec. 4, Roosevelt Rd. 10764, Taipei, Taiwan, R. O. C.
}

\begin{abstract}
A sliding observer for induction machines is designed by use of sliding mode approach. The observation is based on measurements of stator voltages, currents, and shaft angle. The observer is equipped with a switching feedback action to cope with the nonlinearities, input disturbances, and some uncertainties in machine dynamics. A linear continuous feedback is used to characterize the convergence of observation error which is proven to be asymptotically stable.

I. Introduction

The presence of more effective control methods like fieldorientation control [2] and feedback linearization. [8] has made it possible that dc machines can be replaced by inauction machines in various servo systems. Induction machines are preferred because of their less cost, compact size, convenience in maintenance, and high reliability. But on-line information of machine states like rotor flux or rotor current are essential in those sophisticated control methods either for control feedback or for parameter adaptation $[1,9]$. To provide such information by directly attaching sensors to the motor is expensive and unreliable $[2,6]$. In this paper we design a sliding observer to deal with the estimation problem based only on explicitly measurable quantities of stator voltages, currents, and shaft angle. Nonlinearities, model uncertainties, and parameter variations are the difficulties to be faced.
\end{abstract}

Most observer designs for induction machines published in the literatures $[6,7]$ treated the electrical part of machine dynamics separately and the rotor speed was regarded as an available time-varying parameter. Sensitivities of estimation errors to parameter uncertainties and divergence caused by forward Eular approximation were reported therein. On the other hand, nonlinear observers [4] can also be applied to solving such an estimation problem. An example is that Atkinson et al. [11] employed an extended Kalman filter to perform simultaneous estimations of induction motor states and parameters. Our work here adopts a quite different methodology called sliding mode approach $[3,5,10]$

With a hypersurface being defined as a function of the observation errors, sliding conditions are deterr: that the manifold can be reached in finite time. Once the error trajectories have been on the hypersurface, an error based chattering action keeps them sliding along the surface and asymptotically converging to zero. Like sliding mode control, sliding observers are expected to exhibit good robustness to parametric uncertainty and can easily cope with the nonlinearity in machine dynamics $[5,10]$. II. Problem Formulation

The dynamics of a three-phase induction motor can be represented by a convenient 2 -axis model defined in the stator-fixed frame [9]

$$
\mathbf{L} \underline{\dot{x}}+\mathbf{C}(\underline{x}) \underline{x}+\mathbf{R} \underline{x}=\underline{u}
$$

where

$$
\begin{aligned}
& \underline{x} \triangleq\left[\begin{array}{lllll}
i_{s x} & i_{s y} & i_{r x} & i_{r y} & \omega_{r}
\end{array}\right]^{T}, \underline{u} \triangleq\left[\begin{array}{lllll}
v_{s x} & v_{s y} & 0 & 0 & \frac{-2 \tau_{l}}{3 n}
\end{array}\right]^{T},(2) \\
& \mathbf{L} \triangleq\left[\begin{array}{ccc}
L_{s} \mathbf{I}_{2} & L_{m} \mathbf{I}_{2} & 0 \\
L_{m} \mathbf{I}_{2} & L_{r} \mathbf{I}_{2} & \mathbf{0} \\
0 & 0 & 2 J / 3 n
\end{array}\right] \\
& \mathbf{C}(\underline{x}) \triangleq\left[\begin{array}{cc}
\mathbf{C}_{11} & -\underline{c}_{12} \\
\underline{c}_{12}^{T} & 0
\end{array}\right], \mathbf{C}_{11} \triangleq-\omega_{\tau}\left[\begin{array}{cc}
L_{2} \mathbf{J}_{2} & L_{m} \mathbf{J}_{2} \\
L_{m} \mathbf{J}_{2} & L_{r} \mathbf{J}_{2}
\end{array}\right](4)
\end{aligned}
$$

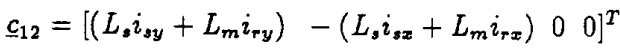

$$
\begin{aligned}
& \mathbf{R} \triangleq \operatorname{diag}\left(R_{s}, R_{s}, R_{r}, R_{r}, \frac{2 b}{3 n}\right)
\end{aligned}
$$

with $\mathbf{I}_{2}$ being the $2 \times 2$ identity matrix and $\mathbf{J}_{2} \triangleq\left[\begin{array}{cc}0 & -1 \\ 1 & 0\end{array}\right]$ The notations are similar to those in [9]. The term $\mathbf{C}(\underline{x}) \underline{x}$ in model (1) forms the nonlinearity of machine dynamics. Besides, there are significant variations of $\mathbf{R}_{\mathbf{r}}$ and $\tau_{l}$ during operation and they are generally assumed uncertain. Our objective is to design a sliding observer of the form

$$
\mathrm{L} \underline{\dot{\hat{x}}}+\mathbf{C}(\underline{\hat{x}}) \underline{\hat{x}}+\mathbf{R} \underline{\hat{x}}=\underline{\hat{\hat{x}}}+\underline{f}(\underline{\hat{x}}, \underline{\tilde{y}})
$$

which will make the observation error $\underline{\tilde{x}}$ converges to zero asymptotically in spite of the nonlinearity and uncertainty. The symbols like $\hat{\mathbf{R}}$ and $\underline{\hat{x}}$ denote the nominal or estimated values of parameters or state variables and the ones like $\underline{y}$ represent the deviations from the actual values where $\underline{\tilde{y}} \triangleq$ $\underline{y}-\underline{\hat{y}}$. The measurable outputs are defined as

$$
\underline{y}=\mathbf{H} \underline{x}=\left[\begin{array}{lll}
i_{s x} & i_{s y} & \omega_{r}
\end{array}\right]^{T}
$$

\section{Sliding Observers}

The error feedback vector function $f(\cdot, \cdot)$ in observer model (7) comprises two parts, one for linear feedback and the other for switching action. It is determined by use of Lyapunov stability analysis. With $\mathbf{L}$ and $\mathbf{R}$ being positive symmetric, 
$\mathrm{C}(\cdot)$ being skew-symmetric, and the assumption that there exists a vector $\zeta[3]$ such that

$$
-\tilde{\mathbf{R}} \underline{x}+\underline{\tilde{u}}=\mathbf{H}^{T} \zeta,\|\zeta\| \leq \rho,
$$

and the bound $\rho$ is known or can be estimated, we construct the following feedback law

$$
\underline{f}(\underline{\hat{x}}, \underline{\tilde{y}})=\mathbf{K} \underline{\tilde{y}}+\mathbf{K}_{\Pi}(\underline{\hat{x}}) \underline{\tilde{y}}+\underline{s}(\underline{\tilde{y}}) \rho
$$

where $\mathbf{K}$ is designed to make the matrix $-(\hat{\mathbf{R}}+\mathbf{K H})$ negative definite, $\mathbf{K}_{C}(\underline{\hat{x}})$ is solved from the equation $\Pi(\underline{\hat{x}})+\mathbf{K}_{\Pi}(\underline{\hat{x}}) \mathbf{H}=$ 0 with $\mathbf{C}(\underline{\tilde{x}}) \underline{\hat{x}}=\Pi(\underline{\hat{x}}) \underline{\tilde{x}}$, and the switching action

$$
\underline{s}(\underline{\tilde{y}}) \triangleq \begin{cases}\|\underline{\tilde{y}}\|^{-1} \mathrm{H}^{T} \underline{\bar{y}}, & \|\tilde{\tilde{y}}\| \neq 0 \\ 0, & \|\underline{\tilde{y}}\|=0\end{cases}
$$

It can be shown that the observation error $\underline{\tilde{x}}$ will die out asymptotically. In general, the shaft speed is measured by use of a techometer or approximated by the difference of angles obtained from encoders. The result is somewhat contaminated by noises. To estimate rotor speed, we use one more differential equation $\dot{\theta}_{r}=\omega_{r}$ and the corresponding observer equation

$$
\dot{\hat{\theta}}_{\tau}=\hat{\omega}_{r}+k_{\theta_{r}} \tilde{\theta}_{r}+\rho_{\theta_{r}} \cdot \operatorname{sgn}\left(\tilde{\theta}_{r}\right)
$$

If the sliding surface $\theta_{r}=0$ have been reached in finite time, we obtain $[5$

$$
\tilde{\omega}_{r}=\rho_{\theta_{r}} \cdot \operatorname{sgn}\left(\tilde{\theta}_{r}\right)
$$

An observation error feedback can thus be realized by use of $k_{\omega} \rho_{\theta_{r}} \cdot \operatorname{sgn}\left(\tilde{\theta}_{r}\right)$. With the switching action $\underline{s}(\underline{\tilde{y}})$ being modified and similar assumption about uncertainties, the observer will estimate the exact state asymptotically.

\section{Conclusion}

The sliding observer presented in this paper is intended for coping with the nonlinearities and some uncertainties in induction machine models. Only minor modifications are needed when other state representation like flux vector is preferred. Part of the simulation result is shown in the figure. The estimation is good in the presence of $20 \%$ error of all nominal parameters and an unknown loading torque, with $4 \mathrm{KHZ}$ sampling, bounded-layer smoothing and backdifference approximation. The influence of measurement noises, simplifying of modeling, and other uncertainties is to be examined by more simulation or real implementation in further investigation. Another directions of further research are to relax the assumption of uncertainty properties in induction machines and to include parameter adaptation mechanism.

\section{Acknowledgement}

The research with this paper is sponsored by National Science Council under the pivjciv numbered NSC-81-0404-E-002-019. The authors would like to thank the foundation for its financial support.

\section{References}

[1] M. Vidyasagar, "On the Stabilization of Nonlinear Systems Using State Detection," IEEE Trans. Automat. Contr., Vol. AC-15, No.3, 1980.
[2] W. Leonhard, "Microcomputer Control of High-Performance Dynamic AC Drives: A Survey," Automatica, Vol. 22, No. 1, pp.1-19, 1986.

[3] B.L. Walcott and S.H. Żak, "Observation of Dynamical System in the Presence of Bounded Nonlinearities/ Uncertainties," Proc. IEEE CDC, pp. 961- 966, 1986.

[4] B.L. Walcott, M.J. Corless and S.H. Żak, "Comparative Study of Nonlinear State-Observation Techniques," Int. J. Control, Vol. 45, No.6, pp. 2109-2131, 1987.

[5] J.-J. E. Slotine, J.K. Hedrick and E.A. Misawa, " On Sliding Observers for Nonlinear Systems," Trans. of ASME Journal of Dynamic Systems, Measurement, and Control, Vol. 109, pp. 245-252., 1987.

[6] G.C. Verghese and S.R. Sanders, "Observers for Flux Estimation in Induction Machines," IEEE Trans. on Industrial Electronics, Vol. 35, No.1, pp. 85-94, 1988.

[7] A. Bellini, "Analysis and Design of a Microcomputer-based Observer for an Induction Motor", Automatica, Vol. 24, No. 4, pp. 549-555, 1988.

[8] R. Marino, S. Peresada and P. Valigi, "Adaptive Partial Feedback Linearization of Induction Motors," Proc. IEEE $C D C$, pp. 3313-3318, December 1990.

[9] R. Ortega, "An Exponentially Convergent Controller for Induction Motors," Proc. ECC91, pp. 7-12, July 1991.

[10] C. Canudas De Wit and J.-J. E. Slotine, " Sliding Observers for Robot Manipulators," Automatica, Vol. 27, No.5, pp. 859-864, 1991.

[11] D.J. Atkinson, P.P. Acarnley, and J.W. Finch, "Observers for Induction Motor State and Parameter Estimation," IEEE Trans. on Industrial Applications, Vol. 27, No. 6, pp. 1119$1127,1991$.

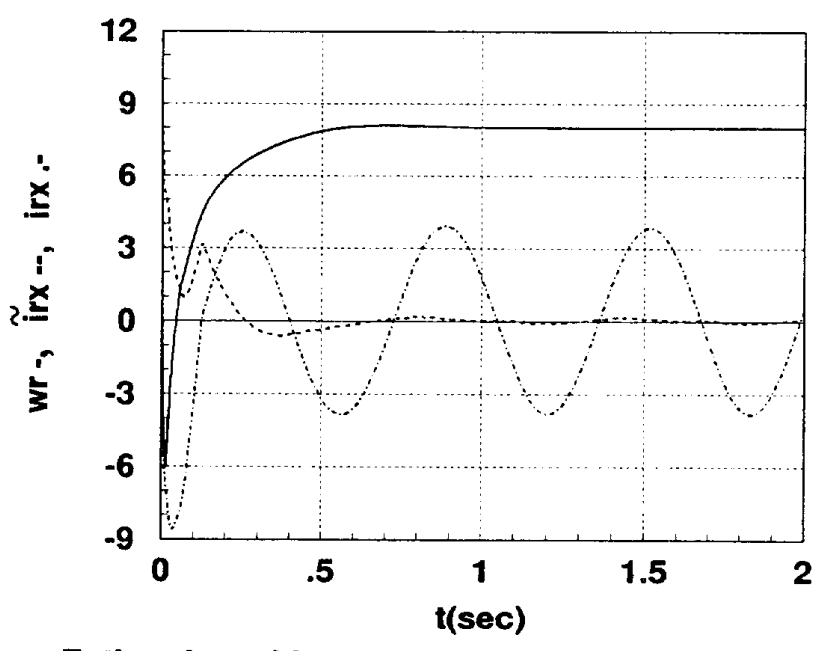

Estimation with parametric uncertainties 